\title{
Kernos
}

Revue internationale et pluridisciplinaire de religion grecque antique

19 | 2006

Varia

\section{La «Chronique de Lindos » : un catalogue à la gloire du sanctuaire d'Athéna Lindia}

\section{Natacha Massar}

\section{(2) OpenEdition \\ Journals}

Édition électronique

URL : https://journals.openedition.org/kernos/452

DOI : $10.4000 /$ kernos.452

ISSN : 2034-7871

\section{Éditeur}

Centre international d'étude de la religion grecque antique

\section{Édition imprimée}

Date de publication : 1 janvier 2006

Pagination : 229-243

ISSN : 0776-3824

\section{Référence électronique}

Natacha Massar, «La " Chronique de Lindos » : un catalogue à la gloire du sanctuaire d'Athéna Lindia », Kernos [En ligne], 19 | 2006, mis en ligne le 22 mars 2011, consulté le 24 août 2022. URL http://journals.openedition.org/kernos/452 ; DOI : https://doi.org/10.4000/kernos.452 


\title{
La «Chronique de Lindos » : un catalogue à la gloire du sanctuaire d'Athéna Lindia*
}

\begin{abstract}
Résumé : L'analyse de la sélection et de l'organisation des notices de la Chronique (dons et épiphanies) au travers des dédicants permet de mieux comprendre la signification de ce document, le public auquel il était destiné, et la culture de ses auteurs. La perspective de cette œuvre est locale, reflétant l'histoire de Lindos - cité-état puis dème -, de son sanctuaire, et le rôle croissant de ses prêtres.
\end{abstract}

Abstract: The "Chronicle of Lindos": A catalogue to the glory of Athena's sanctuary. An analysis of the selection and the organisation of the Chronicle (gifts and epiphanies) based on the identity of the donors offers an understanding of the significance of the document, the public it is addressed to, and the culture of its authors. The work presents a local perspective which reflects the history of Lindos, City-State then deme, of is sanctuary, and of the increasing importance of its priests.

Partant de la définition d'un catalogue comme «liste sélective organisée selon un ordre qui lui donne un sens propre», je voudrais entreprendre l'analyse d'une ouvre, la «Chronique de Lindos ». Ce document est gravé sur un bloc de $2,37 \mathrm{~m}$ de haut et $0,85 \mathrm{~m}$ de large, qui se dressait dans le sanctuaire d'Athéna à Lindos, sur l'île de Rhodes. La stèle est gravée sur sa face principale et le texte est réparti en quatre zones distinctes. Au sommet, couvrant toute la largeur de la pierre, figure le décret qui stipule l'érection du monument ainsi que la rédaction et la gravure du document qui suit. Ce second texte, réparti en trois colonnes, comprend deux parties : les données mémorables concernant les dédicaces à Athéna Lindia et les épiphanies de la déesse ${ }^{1}$.

Le décret, daté par le prêtre éponyme d'Athéna Lindia de 99 av. J.-C., apporte quelques informations sur les motivations qui ont poussé les Lindiens à faire rédiger la «Chronique ». Les considérants évoquent en effet l'âge

\footnotetext{
* Les commentaires d'Alexis d'Hautcourt et de Vinciane Pirenne-Delforge à propos d'une version antérieure de cet article m'ont été précieux, et je les en remercie vivement. Durant l'année académique 1999-2000, D. Viviers a dirigé à l'Université libre de Bruxelles un séminaire consacré aux dédicaces dans les sanctuaires, en particulier ceux de Délos et de Lindos, qui a inspiré plusieurs réflexions développées ici.

${ }^{1}$ Éditions de l'inscription : Chr. Blinkenberg, Lindos. Fouilles de l'Acropole 1902-1914. II Les Inscriptions, Berlin / Copenhague, 1941; Anagraphe von Lindos, 532 F 1 (éd. JACOBY); C. HiGBIE, The Lindian Chronicle and the Greek Creation of their Past, Oxford, 2003, p. 18-49 (texte grec avec trad. anglaise); présentation du contenu du décret et de la Chronique (avec une liste des dédicants et des offrandes) dans J. SHAYA, «The Greek Temple as Museum: The Case of the Legendary Treasure of Athena from Lindos », AJA 109 (2005), p. 437-439. Les quatre parties sont désignées par les lettres A (décret), B-C-D (colonnes).
} 
vénérable du sanctuaire (A 1. 2), et la beauté des dédicaces détruites par le temps (A 1. 4). Ils associent les offrandes aux « épiphanies de la déesse » (A 1. 3), et précisent que les auteurs pressentis rédigeront ce qu'ils jugent convenable au sujet des offrandes et des apparitions en se fondant sur les documents disponibles. Le décret précise les noms des deux auteurs de la Chronique, Tharsagoras, fils de Stratos, de Ladarma et Timachidas, fils d'Hagesitimos, de Lindos, qui est le fils du proposant du décret².

L'étude qui suit se concentrera sur la première partie de la "Chronique »" à savoir les offrandes mémorables dédiées à Athéna Lindia. Mais s'agit-il réellement d'un catalogue au sens où ce terme est défini ci-dessus ? La sélection opérée par Tharsagoras et Timachidas est évidente, en particulier pour la période la plus récente : n'ont été mentionnés que les dons par trois instances, le dème de Lindos, la cité de Rhodes et des souverains grecs ${ }^{4}$. Qu'ils ont proposé leur propre organisation des dédicaces apparaît au regard des sources qu'ils citent après chaque notice : ils n'ont pas suivi une source unique, mais ont réarrangé à leur manière les données trouvées chez d'autres ${ }^{5}$. La «Chronique » a fait l'objet de nombreux commentaires, concernant en particulier les objets dédiés ou les nombreuses sources citées, mais sa structure n'a jamais été analysée en détails. Or, une telle étude permet de revenir sur des questions qui ont récemment fait l'objet d'hypothèses controversées ${ }^{6}$ : quel est le sens général du document? Quels sont les buts poursuivis par les auteurs du texte ? À quel public le monument était-il d'abord destiné ?

Cette analyse vise à mettre en évidence la logique interne de ce catalogue, à comprendre la sélection et l'ordre des notices, en privilégiant un terme d'analyse, à savoir les dédicants. Ce choix est dicté par le document luimême : la première partie de la Chronique est introduite par le titre «Voici

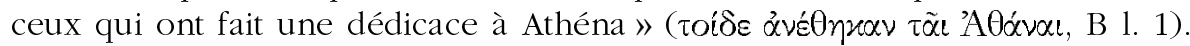
Et, conformément à cette logique, le nom du dédicant figure toujours en tête des notices. Leur identité semble donc cruciale à la compréhension et

\footnotetext{
${ }^{2}$ Timachidas est cité ailleurs, comme grammairien notamment, ce qui a poussé des auteurs modernes à le considérer comme seul véritable auteur de la «Chronique ». On peut se demander pourquoi le décret, proposé par le père de Timachidas, mentionnerait un autre auteur que son fils, s'il n'avait pas une part reconnue de responsabilité dans le texte. Voir A. Chaniotis, Historie und Historiker in den griechischen Inschriften, Stuttgart, 1988, p. 56 et 127-128; sur les auteurs, voir HiGBIE, o.c. (n. 1), p. 62-3.

3 La dénomination «Chronique », proposée par l'inventeur de l'inscription, Chr. Blinkenberg, a souvent été dénoncée car elle désigne un texte historique à forte structure chronologique, ce qui n'est pas le cas ici. Cependant, définir ce document par un seul terme est difficile, et la plupart des auteurs adoptent le titre de Blinkenberg. Voir F. JACOBY, Die Fragmente der griechischen Historiker, IIIb Kommentar, Leyde, 1955, p. 445; CHANIOTIS, o.c. (n. 2), p. 53.

${ }^{4}$ CHANIOTIS, o.c. (n. 2), p. 54

${ }^{5}$ Voir par ex. SHAYA, l.c. (n. 1), p. 428 et n. 27.

${ }^{6}$ HigBie, o.c. (n. 1) n. 2 p. 204 et 291-3 considère que la chronique est destinée surtout à un public de visiteurs (touristes) étrangers, une idée inspirée de l'œuvre de Pausanias. Voir aussi SHAYA, l.c. (n. 1), p. 435-6. Cette insistance sur Pausanias est remise en question dans le compte rendu de J. MARINCOLA, http://ccat.sas.upenn.edu/bmcr/2005/2005-04-59.html.
} 
l'interprétation de l'œuvre, et elle servira de fil conducteur à cette étude? Dans l'esprit des rédacteurs, les épiphanies forment un tout avec les dédicaces, et elles seront donc également envisagées afin de tenter d'élucider la logique globale de l'œuvre.

\section{La structure générale du catalogue d'offrandes}

La mise en place graphique de la «Chronique » établit une séparation nette entre les dédicaces et les épiphanies : chaque partie est introduite par un titre, et les récits d'épiphanies occupent une colonne à part. Les notices sont bien isolées : chaque apparition est précédée d'un sous-titre, alors que les dédicaces débutent toujours sur une nouvelle ligne et sont séparées les unes des autres par un trait horizontal. Cependant, aucune sous-partie n'est indiquée : le catalogue apparaît comme un tout constitué d'une succession de notices $^{8}$. Cette absence de divisions explicites laisse la porte ouverte à de multiples lectures. J'en propose une ici qui ne se veut pas exclusive, mais complémentaire à d'autres approches.

La structure de la chronique est globalement chronologique, allant des temps mythiques jusqu'à une époque proche des rédacteurs, indéterminée car les notices les plus récentes sont perdues. L'ensemble se structure en trois parties, mythique ( $\left.n^{\circ} 1-14\right)$, archaïque/classique $\left(\mathrm{n}^{\circ} 15-33\right)$, IV $\mathrm{IV}^{\mathrm{e}}$ siècle hellénistique ( $\left.n^{\circ} 34-42\right)$. Chacune est divisée en deux sous-sections : la première comprend des dédicaces par des Lindiens (ou des Rhodiens) et la seconde par des étrangers'. Chaque partie commence par la dédicace d'un individu ou d'un groupe lindien, dont l'identité signale une rupture avec ce qui précède.

La toute première offrande a été faite par le héros éponyme Lindos; elle est suivie de celle des Telchines, des figures mythiques étroitement associées à Rhodes; ensuite, de Kadmos à Teucros, les dédicants sont des étrangers. Vient ensuite le début de la période civique avec une dédicace par les phylai

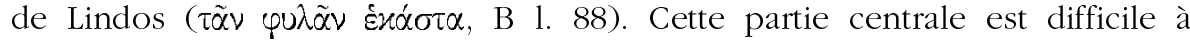
cerner, car très mal conservée : des notices 18 à 22 il ne reste presque rien. Cependant, le texte préservé (les $n^{\circ} 15$ à 17 , puis les $n^{\circ} 23$ à 33) respecte la répartition Lindiens - étrangers. Un indice supplémentaire tend à confirmer cette organisation : en effet, comme à la période suivante, lorsque l'on passe de la dernière notice "lindienne" au premier don par un étranger, on retourne en arrière dans le temps. Ainsi, la notice 23 mentionne ceux qui accompagnaient le tyran Kléoboulos lors d'une expédition contre les Lyciens

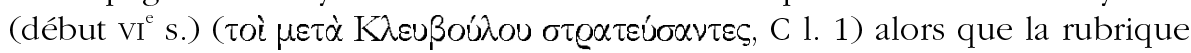

\footnotetext{
${ }^{7}$ Voir à ce sujet le commentaire de SHAYA, l.c. (n. 1), p. 429

${ }^{8}$ Chaque notice présente les mêmes éléments dans le même ordre : dédicant puis objet dédié (avec parfois des précisions sur les circonstances, le matériau, les inscriptions qu'il porte), les sources où les auteurs ont puisé les informations et leurs éventuelles discordances.

${ }^{9}$ Je suis le découpage proposé par Chaniotis, o.c. (n. 2), p. 55, qui, le premier, a identifié cette répartition récurrente entre Lindiens et non Lindiens. Je considère cependant qu'il y a trois grandes périodes subdivisées en Lindiens / non Lindiens plutôt que six parties distinctes.
} 
24 commémore une victoire des gens de Phaselis, menés par l'oikistès Lakios, contre les Solymoi. Or Phaselis fut fondée en 691: on revient donc en arrière d'environ un siècle ${ }^{10}$.

La troisième et dernière partie est introduite par une dédicace des Lindiens ( $\Lambda$ ívôıo ; $\mathrm{n}^{\circ} 34$ ) après une série de dons par des étrangers. Dans les quatre premières notices alternent des offrandes des Lindiens et du damos (de Rhodes); elles sont suivies de dédicaces par un groupe précis d'étrangers, des souverains grecs. Cette répartition conduit ici aussi à un hiatus chronologique: la dernière offrande du damos se situe lors d'une guerre contre Ptolémée II Philadelphe; elle est donc plus récente que la première offrande par un souverain, à savoir Alexandre le Grand.

Quelles sont les limites chronologiques entre les trois périodes? On reconnaît bien d'abord les temps mythiques allant jusqu'au retour des héros de Troie. La première notice de la deuxième partie donne le ton de cette section : il s'agit de dédicaces par les phylai, en d'autres termes des subdivisions de Lindos, cité indépendante. Quel incident a déterminé la répartition entre la $2^{\mathrm{e}}$ et la $3^{\mathrm{e}}$ période ? Autour de 400, deux événements marquent Lindos et son sanctuaire à vingt ans d'intervalle : le synécisme de Lindos, Ialysos et Camiros en 408/7 et l'incendie du temple d'Athéna en 392/1 ${ }^{11}$. Il est reconstruit sans doute quelques dizaines d'années plus tard; ce dernier bâtiment est toujours debout à l'époque où est rédigée la «Chronique ». Dans la perspective des auteurs, à savoir une glorification du sanctuaire d'Athéna, le choix du synécisme comme date charnière semble particulièrement peu heureux : dorénavant, le culte d'Athéna n'est plus un culte poliade, Lindos n'étant «plus que » un dème de la cité de Rhodes. En revanche, la reconstruction du temple marquerait une articulation chronologique positive ${ }^{12}$. Un indice pourrait confirmer cette interprétation : le premier don de la dernière période consiste en parures pour la statue de la déesse, ce qui pourrait constituer la touche finale de l'ornementation du nouveau temple ${ }^{13}$.

Ces grandes subdivisions étant établies, revenons plus en détail à chaque période, en nous attardant sur les critères de choix et sur certains éléments d'organisation. Lorsque l'on peut établir un lien entre une notice et une épiphanie, ce sera précisé afin d'éclairer la structure globale de l'œuvre.

${ }^{10}$ Sur ces deux notices, et l'ordre anti-chronologique dans lequel elles apparaissent, voir A. Bresson, "Rhodes and Lycia in Hellenistic Times ", in V. GABrielsen et al. (éds), Hellenistic Rhodes: Politics, Culture, and Society, Aarhus, 1999, p. 102

${ }^{11}$ Voir un résumé de la discussion sur la date et les références chez Chr. HABICHT, « Rhodian Amphora stamps and Rhodian Eponyms », REA 105 (2003), p. 542, n. 3.

${ }^{12}$ Cette hypothèse fut déjà proposée par JACOBY, o.c. (n. 1), p. 449; il considère cependant, à la suite de Blinkenberg, que la notice 34 (première dédicace des Lindioi) doit être rattachée à la période archaïque et non à la $3^{\mathrm{e}}$ période.

${ }^{13}$ Voir HigBIE, o.c. (n. 1), p. 128. 


\section{Première période : les temps mythiques, géographie et cultes}

Après Lindos et les Telchines, figures de la haute antiquité lindienne et rhodienne, viennent trois grandes figures panhelléniques, Cadmos, Minos, Héraclès $(3,4$ et 5$)$. Leur ordre de succession suit une chronologie acceptée si l'on se réfère au Marbre de Paros qui les présente dans le même ordre en appuyant cet enchaînement sur des dates ${ }^{14}$. À Héraclès succèdent des participants à la guerre de Troie. Par les objets qu'il dédie, le héros sert de charnière entre ces deux groupes. Il offre en effet deux boucliers inscrits, celui d'Eurypylos pris aux Méropes (les habitants de Cos), et celui de Laomédon, roi de Troie. Or, les épisodes évoqués par ces noms sont liés, mais surviennent dans l'ordre chronologique inverse. Héraclès, suite à une traitrise de Laomédon, retourne avec une petite troupe pour se venger : c'est la première Guerre de Troie. Lors du voyage de retour, il échoue sur l'île de Cos où il combat les Méropes. Ainsi, ces hauts faits d'un héros «panhellénique » introduisent déjà le conflit troyen.

Le dédicant qui suit immédiatement Héraclès est son fils, Tlépolème (6), «fondateur politique» de Rhodes et héros homérique ${ }^{15}$. Une succession généalogique signale le passage du temps; un autre fils du même héros, Téléphos, apparaît à la notice 8 . Le déroulement de la guerre de Troie est suggéré par des notations circonstancielles. Ainsi, Tlépolème, qui mène le contingent rhodien à Troie ${ }^{16}$, fait une dédicace avant son départ. Les troupes qui l'accompagnaient font un don à la déesse à leur retour, pour commémorer leur victoire. Entre ces deux notices (6 et 9) se déroule le long conflit où Tlépolème trouve la mort. Les dédicaces suivantes, par Ménélas, Hélène et leur gubernétès Canopos (10-12), évoquent également le retour de Troie. La dédicace 14, qui clôt cette période, a été faite par Teucros. Ce personnage est le fils de Télamon, compagnon d'Héraclès lors de son raid contre Troie, et d'Hésionè, la fille de Laomédon. Par ses ascendants, il rappelle donc l'expédition troyenne d'Héraclès évoquée par les offrandes du fils de Zeus, fermant la «boucle troyenne».

Que peut-on dire de cette sélection des héros et figures légendaires ? Par leur biais, Lindos peut se rattacher à de grands cycles mythiques panhelléniques. Les gestes de héros permettent en effet aux cités grecques d'invoquer les antécédents mythiques de relations historiques, dans le cadre de reconstructions de leur passé ou de négociations diplomatiques ${ }^{17}$. Ainsi, les voyages de Cadmos le Phénicien permettent à de nombreuses cités, dont Rhodes, de

\footnotetext{
${ }^{14}$ Marm. Par., 239A 7, 11, 18 (éd. JACOBY). Ce rapprochement a déjà été opéré par BuNKENBERG, o.C. (n. 1), col. 162.

${ }^{15}$ Sur les divers fondateurs de Rhodes (et Lindos), et le rôle "politique » de Tlépolème, voir I. MALKIn, La Méditerranée spartiate, Paris, 1999, p. 54-55.

${ }^{16}$ Homère, Iliade II, 653-656.

${ }^{17}$ Un domaine beaucoup étudié ces dernières années, notamment par I. Malkin. Les travaux sur les parentés légendaires vont dans le même sens; voir par ex. C.P. Jones, Kinship Diplomacy in the Ancient World, Cambridge, Mass. / Londres, 1990.
} 
se donner une antiquité phénicienne ${ }^{18}$; Héraclès rappelle toute la Grèce «dorienne ». En outre, certains personnages sont associés à des régions qui ont joué un rôle dans l'histoire lindienne: Minos représente la Crète, qui réapparaîtra dans des contextes conflictuels ou commerciaux ${ }^{19}$; Canopos, le pilote de navire de Ménélas (12), évoque l'Égypte où il est mort. Ce pays restera en relation avec Rhodes aux époques suivantes (dons d'Amasis et mentions de rois Lagides) ${ }^{20}$. Parfois, les indications géographiques sont plus spécifiques. Selon la notice 8 , Téléphos a fait une dédicace suite à un oracle d'Apollon Lycien, dont le sanctuaire, situé à Patara sur la côte lycienne, aurait été fondé selon certaines traditions par un des Telchines. Dans cette cité on vouait un culte à Téléphos ${ }^{21}$. Cette notice établit donc un lien direct entre Lindos et la Lycie, la région côtière qui lui fait face, avec laquelle elle aura des relations historiques mentionnées plus loin. Seule la Sicile, très présente à l'époque archaïque, et rappelée par Hiéron de Syracuse à la période hellénistique, n'est pas évoquée à travers une figure mythique.

Un autre aspect, bien plus difficile à cerner, concerne le lien entre ces figures mythiques et la vie cultuelle lindienne ${ }^{22}$. En effet, nombre de ces dédicants font l'objet d'un culte à Lindos ou quelque part à Rhodes, sans que l'on puisse en déterminer la localisation exacte : il en va ainsi de Lindos à qui était dédié un héroon ${ }^{23}$, d'Héraclès ${ }^{24}$; de Tlépolème (des Tlépolémaia sont attestés dès le $\mathrm{v}^{\mathrm{e}} \mathrm{s}$. $)^{25}$, d'Hélène ${ }^{26}$. Téléphos a fondé le sanctuaire de Poséidon à Ialysos, autre cité rhodienne, mais aucun culte qui lui serait lié n'est attesté à Lindos. A. Bresson a toutefois suggéré que la dédicace du héros, faite à l'injonction d'Apollon lycien, pourrait signaler un lien entre ce culte et celui d'Athéna Lindia ${ }^{27}$.

18 Sur Cadmos, archétype du voyageur, associé à des « origines phéniciennes », voir MALKIN, o.c. (n. 15), p. 119-120. Voir aussi HigBIE, o.c. (n. 1), p. 215.

1916 : dékatè du bateau de Crète; 34 : dékatè du butin pris aux Crétois. Rhodes est en conflit avec la Crète, ostensiblement pour combattre la «piraterie crétoise ", lors des première et seconde guerres crétoises en 205-4 et en 155-3. Voir par ex. V. GABRIELSEN, The Naval Aristocracy of Hellenistic Rhodes, Aarhus, 1997, p. 45; P. PERLMAn, « Kretes aiei leistai? The Marginalization of Crete in Greek Thought and the Role of Piracy in the Outbreak of the First Cretan War ", in GABRIELSEN et al. (éds), o.c. (n. 10), p. 132-161.

${ }^{20} \mathrm{n}^{\circ} 29,37,39$ et $3^{\text {e }}$ épiphanie. HigBie, o.c. (n. 1), p. 89-90 sur Canopos. Sur les relations diplomatiques et commerciales entre Lindos, Rhodes et l'Égypte, voir GABRIELSEN, o.c. (n. 19), p. $71-4$.

${ }^{21}$ BlinkenberG, o.c. (n. 1), 274; 282 1. 31; 294 1. 22; 299 1. C16; 317 1. A28-9 et B.87-8; 3491. 22-3; 378 1. B54, 409 1. 3. Voir HigBie, o.c. (n. 1), p. 84 et Bresson, l.c. (n. 10), p. 100.

${ }^{22}$ Sur les autres divinités qui sont honorées avec Athéna par les dédicants de la Chronique, voir HigBIE, o.c. (n. 1), p. 179-180 (Zeus, Poséidon et Héraclès).

${ }^{23}$ HigBie, o.c. (n. 1), p. 63-64; SHAYA, l.c. (n. 1), n. 65 p. 435.

${ }^{24}$ HigBIE, O.C. (n. 1), p. $70-71$

${ }^{25}$ Pindare, Olympique VII, 77-81. Voir aussi A. BRESSON, Recueil des inscriptions de la Pérée rbodienne, Paris, 1991, $\mathrm{n}^{\circ} 5,1.8$.

${ }^{26}$ Pausanias, III, 19, 9-10. HigBIE, o.c. (n. 1), p. 217-218.

${ }^{27}$ BRESSON, l.c. (n. 10), p. 100. 


\section{Deuxième période : les cité de Lindos et ses colonies}

L'analyse de la première section de cette période est rendue difficile par son mauvais état de conservation et seules des observations ponctuelles sont possibles. La notice $\mathrm{n}^{\circ} 17$ consigne le don des Lindiens qui ont participé à la colonisation de Cyrène avec Battos (B 1. 109-10). Elle évoque déjà ce qui va être le thème majeur de la deuxième section, les colonies, mais en même temps, elle confirme l'hypothèse de la répartition entre Lindiens et étrangers. En effet, le premier mot de la notice est $\Lambda \iota \delta ́ i ́ \omega v$, insistant sur l'identité lindienne des dédicants. Cette précision contraste avec les notices de la seconde section où c'est toujours l'ethnique de la colonie qui est donné.

La notice 23 (haut de la $2^{\mathrm{e}}$ colonne) évoque une figure vénérable de l'histoire archaïque lindienne, le tyran Kléoboulos. Il fut compté au nombre des Sept Sages et, à ce titre, fait l'objet d'une notice par Diogène Laërce ${ }^{28}$, qui nous livre l'essentiel des informations que l'on possède à son sujet. L'un de ses actes mémorable serait la (re)construction du temple d'Athéna Lindia. Ici, la notice évoque une victoire militaire dans une région déjà rencontrée, la Lycie. La dédicace est faite non par le tyran lui-même, mais par ceux qui l'accompagnent lors de son expédition: une formulation qui rappelle la dédicace $n^{\circ} 9$ faite par ceux qui accompagnaient Tlépolème à Troie. Or, le don 6 est offert par le héros, et l'on peut se demander si l'une des notices mutilées ne mentionnait pas une dédicace par Kléoboulos en personne.

Dans la deuxième section, les étrangers qui présentent un don à Athéna dessinent une géographie particulière. La première et la dernière notice $\left(\mathrm{n}^{\circ} 24\right.$ et 33) concernent les cités de Phaselis (Lycie) et Soloi (Cilicie), deux colonies lindiennes. Six des huit dédicants qui les séparent viennent de Sicile ou d'Italie du Sud, pour la plupart de cités colonisées par les Lindiens seuls ou en collaboration ${ }^{29}$. Certaines cités, comme Gela et Akragas, apparaissent deux fois ( 25 et 28,27 et 30 ). Au centre de la section figure la notice consacrée à Amasis roi d'Égypte (29). L'avant dernière dédicace (32) fut consacrée par un Perse dont le nom est perdu. Cette section évoque donc une géographie coloniale dont l'unité est brisée par deux exceptions sur lesquelles il faut s'arrêter un instant.

La notice la plus longue du catalogue (19 lignes) est consacrée à la dédicace d'Amasis. En voici le début : «Amasis, roi des Égyptiens, une cuirasse en lin dont chaque fil est constitué de 360 brins, comme en témoigne Hérodote

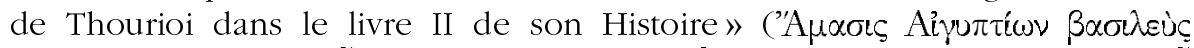

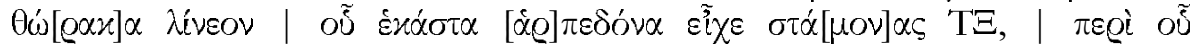

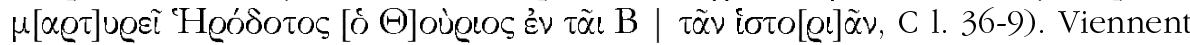
ensuite d'autres sources dont certaines mentionnent des dons supplémentai-

\footnotetext{
${ }^{28}$ Diogène Laërce, I, 89-93 (reconstruction du temple : 89).

${ }^{29}$ L'incertitude porte sur le $\mathrm{n}^{\circ} 26$ dont les dédicants viennent de Sybaris qui, selon Strabon, VI, 1, 14 (C264) est une colonie rhodienne, et sur le $n^{\circ} 31$, très abîmé et dont la restitution est incertaine. Il pourrait cependant s'agir d'un $3^{\mathrm{e}}$ dédicant de Gela; voir HigBIE, o.c. (n. 1), p. 120121.
} 
res : deux statues et des phiales. Ces quatre lignes méritent un commentaire : Tharsagoras et Timachidas ont délibérément choisi d'une part de mettre en évidence cette offrande précise d'Amasis, la plus célèbre dans l'Antiquité - les autres étant évoquées seulement dans un second temps. D'autre part ils ont appuyé leur information sur le seul auteur explicitement non lindien cité dans toute la Chronique ${ }^{30}$. Pour ce faire, ils ont en outre utilisé une formule et un verbe qu'ils n'emploient nulle part ailleurs pour citer un auteur ${ }^{31}$. La notice sur Amasis n'assigne aucune motivation au roi et ne précise pas les circonstances du don. Pourquoi occupe-t-il une telle place dans le catalogue ? La mention d'Hérodote nous fournit un indice : la cuirasse en lin d'Amasis est célèbre audelà des frontières rhodiennes, et ce depuis Hérodote (au moins). La mention de l'ethnique d'Hérodote assure une identification immédiate du père de l'histoire, et révèle en même temps cette renommée internationale. La signification historique des dons n'intéresse guère les auteurs de la Chronique. Ce don est mis en évidence en raison de sa célébrité. Ces deux démarches conjointes, mettre en évidence le renom international d'un don, et appuyer cela par la mention d'une autorité admise de tous, Hérodote, relèvent d'une perception du prestige culturel typiquement hellénistique. Les cités grecques de cette époque se vantent de leurs citoyens savants ou de leurs accomplissements culturels, en particulier s'ils ont acquis une renommée internationale . $^{32}$

La dédicace par un général du roi de Perse a fait couler beaucoup d'encre : s'agit-il d'un don réel ou fictif ? À quelle occasion a-t-il été offert à Lindos? La question est compliquée par le rapport problématique entre cette dédicace et la première épiphanie. Celle-ci relate la manière dont la déesse a sauvé les Lindiens lors du siège de la cité par un navarque de Darius. Très impressionné par cette manifestation divine, le Perse a offert une série d'objets au sanctuaire. La liste de ces dons (D. 1. 35-8) est proche, sans être identique, de celle de la notice 32 , et on a suggéré qu'il s'agissait de deux versions du même événement ${ }^{33}$. Avant de poursuivre l'analyse, signalons d'une part que cette épiphanie, la première, est également de loin la plus longue (58 lignes) et la plus détaillée. D’autre part, le décalage chronologique entre la première dédicace et la première épiphanie est frappant: si l'ancienneté du sanctuaire est attesté par des dons des temps mythiques, les apparitions de la déesse ne commencent, au plus tôt, qu'à la fin du vi iècle, un décalage qu'il faudra tâcher d'élucider.

Pourquoi accorder une telle place aux Perses? Les guerres médiques ont joué un rôle essentiel dans l'imaginaire et la construction identitaire des cités

\footnotetext{
${ }^{30}$ Hérodote, II, 182 et III, 47 (description de la cuirasse de lin). Sur les auteurs non rhodiens cités dans la Chronique, voir JACOBY, o.c. (n. 1), p. 447.

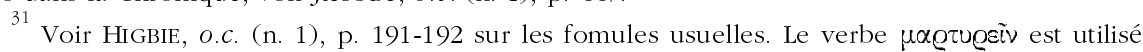
une seule autre fois ( $n^{\circ} 39$, C 1. 113), mais pour citer les archives lindiennes.

${ }^{32}$ Voir N. MASSAR, Soigner et servir. Histoire culturelle et sociale de la médecine grecque à l'époque hellénistique, Bruxelles / Paris, 2005 (Culture E Cité, 2), p. 199-201.

33 BlinkenBerg, o.c. (n. 1), 194-8; HigBie, o.c. (n. 1), p. 124-125.
} 
grecques à l'époque classique - or l'île de Rhodes, sous contrôle perse, se situe en marge du monde grec dans ces conflits ${ }^{34}$. Ces récits signalent clairement que les généraux perses reconnaissent le sanctuaire et, dans le récit d'épiphanie, la puissance d'Athéna. Par là même, ils inscrivent Lindos du «bon côté » dans les rapports entre Grecs et Perses. La multiplication des références aux Mèdes, et la longueur du premier récit d'apparition, révèlent la grande valeur symbolique de ces épisodes.

Les notices de cette période agissent comme un révélateur des réseaux et de l'activité colonisatrice de la Cité de Lindos. Ces relations sont vivantes et dynamiques et les dons s'échelonnent sur deux ou trois siècles. Les colonies reconnaissent et entretiennent leurs relations avec la métropole notamment par des dons au sanctuaire poliade de la cité qui joue un rôle essentiel dans la cohésion de ce réseau ${ }^{35}$. L'origine des dons dessine en filigrane des activités essentielles de ces cités : les plus nombreux ont été faits à la suite d'expéditions militaires $(17,23,24,25,30,33)$; deux d'entre eux à la suite d'un succès commercial (maritime) (16 sans doute, et 26). Pour les dédicants étrangers, en particulier sicéliote, ce sanctuaire représente sans doute aussi un lieu de publicité intéressant, qui touchera un public large appartenant à un réseau qui les intéresse (entre autres pour des raisons commerciales).

Pour la première fois avec le don d'Amasis, on perçoit l'entremêlement entre une réalité archaïque - don d'un puissant souverain étranger qui établit ainsi des liens avec Lindos ${ }^{36}$ - et la perception qu'ont pu en avoir les auteurs hellénistiques de la Chronique, qui n'en retiennent que le prestige conféré au sanctuaire. Enfin, les notices où apparaissent des Perses montrent l'utilisation idéologique que les Lindiens ont pu faire (a posteriori) de la fréquentation de leur sanctuaire poliade. On sort ici du cadre strictement lindien pour entrevoir la manière dont une petite cité grecque a pu chercher à se situer dans un contexte grec plus large par de tels récits.

\section{Troisième période : la montée en puissance des prêtres}

\section{a) Lindos et Rhodes}

La première section de cette période comprend quatre dédicaces alternativement par les Lindiens et par le peuple des Rhodiens ${ }^{37}$. Le contenu de ces dernières évoque des relations internationales : la notice $n^{\circ} 35$ mentionne des objets en or donnés par Artaxerxès au damos (de Rhodes) qui les a ensuite dédiés à Athéna Lindia. Au $n^{\circ} 37$, les Rhodiens, conformément à un oracle, ont offert un bouclier à la déesse pour mettre un terme à la guerre contre Ptolémée II Philadelphe. Cependant, la signification des deux notices est très

\footnotetext{
${ }^{34}$ Ou a un rôle «négatif » : selon Diodore, XI, 3, 8, les Rhodiens auraient fourni des navires à la flotte de Xerxès.

35 A.J. GRAHAM, Colony and Mother City in Ancient Greece, Manchester, 1964.

${ }^{36}$ E.D. FranCis et M. VICKERS, « Amasis and Lindos », BICS 31 (1984), p. 119-130.

${ }^{37}$ Le damos mentionné est bien le peuple des Rhodiens comme en atteste la dédicace sur le

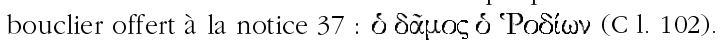


différente : dans le premier cas, Artaxerxès a rendu hommage à la cité de Rhodes qui ensuite, pour une raison inconnue ${ }^{38}$ a offert ses présents au sanctuaire de Lindos. Le second récit en revanche implique directement le sanctuaire lindien dans la politique extérieure rhodienne puisque le don à Athéna doit mettre fin à un conflit avec les Lagides. Cette notice présente donc la déesse comme jouant un rôle essentiel dans le salut des Rhodiens. On la rapprochera du troisième récit d'épiphanie qui concerne également un souverain lagide. Lors du siège de Rhodes par Démétrios, la déesse, apparue en rêve à l'un de ses prêtres, aurait en effet incité les Rhodiens à faire appel à Ptolémée $\mathrm{I}^{\text {er }}$ pour les aider. Dans ce cas également, la déesse lindienne est présentée comme la sauveuse du peuple des Rhodiens.

\section{b) Les souverains}

Dans la dernière section, qui énumère les dons d'étrangers, le critère de sélection des auteurs est clairement mis en évidence : toutes les notices commencent par le titre du dédicant, $\beta \alpha \sigma \iota \lambda \varepsilon u ́ c ̧$. Ces notices sont organisées selon un ordre chronologique. Leur datation révèle une concentration particulière au début de l'époque hellénistique : la dédicace la plus récente conservée fut offerte par Philippe V de Macédoine, sans doute avant 202, date à laquelle Rhodes lui déclare la guerre. Ce roi, pourtant plutôt un ennemi des Rhodiens, figure dans le catalogue au même titre que les autres: c'est clairement son statut qui a déterminé son inclusion dans la série et non des considérations politiques ou diplomatiques relatives à l'histoire de l'île.

Pourquoi Tharsagoras et Timachidas ont-ils opéré cette sélection alors qu'ils appartiennent à une cité dont la neutralité politique et l'indépendance ont été défendues durant des décennies? En faisant ce choix, ils se sont conformés à une conception hellénistique de la culture. Au cours de cette période, les souverains émergent en effet comme les arbitres en diverses matières touchant au culturel : l'attention favorable qu'ils portent à un savant, une œuvre, un sanctuaire, agit comme un révélateur ou un indicateur de son intérêt, et accroît son prestige ${ }^{39}$. Si un sanctuaire est fréquenté par de nombreux rois, cela apparaît comme une preuve irréfutable de son importance ${ }^{40}$. Une autre touche typique de l'époque concerne la notation selon laquelle les armes ont été utilisées par le souverain (C 1. 115 et 122) : un objet offert par le roi acquiert plus de valeur si ce dernier l'a utilisé, une pratique auparavant bien attestée chez les Perses ${ }^{41}$. En outre, pouvant faire eux-mêmes l'objet d'un

\footnotetext{
38 Buinkenberg, o.c. (n. 1), p. 199 a suggéré d'y voir un soutien de la cité de Rhodes à la reconstruction du temple.

${ }^{39}$ MASSAR, O.C. (n. 32), p. 281.

${ }^{40}$ Cela semble exclure que les notices perdues mentionnaient d'autres personnes que des rois, puisque cela signifierait une baisse de prestige.

${ }^{41}$ Le Perse de la $1^{\text {re }}$ épiphanie offre d'ailleurs ce qu'il porte : D 1. 35-6. Pour les rois perses, voir H. SANCISI-WeERdenburg, «Gifts in the Persian Empire », in P. Briant et Cl. Herrenschmidt (éds), Le tribut dans l'Empire perse, Actes de la table-ronde de Paris, 12-13 déc. 1986, Paris / Louvain, 1989, p. 132, 134. Pour les souverains hellénistiques, voir N. MAssAR, «Le rôle des
} 
culte (attesté à Rhodes pour Ptolémée $\left.\mathrm{I}^{\mathrm{er}}\right)^{42}$, ils occupent une sphère particulière, plus proche du divin que les autres hommes.

En revanche, si l'on s'interroge sur les motivations des souverains qui ont offert un don à Athéna Lindia (plutôt que sur les intérêts du sanctuaire), le tableau apparaît sous d'autres couleurs. Les motivations les plus incertaines sont celles d'Alexandre. Chr. Blinkenberg ${ }^{43}$ a suggéré qu'Alexandre a pu faire ce don à l'imitation des rois perses, une hypothèse intéressante surtout à la lumière de la dédicace. Il a en effet offert un sacrifice à Athéna «après avoir

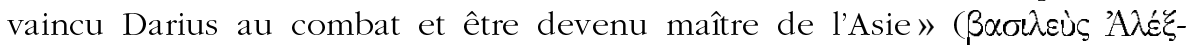

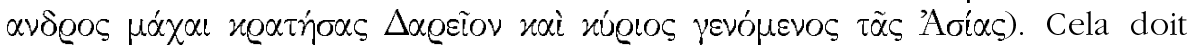
cependant rester une hypothèse. En revanche, les objets dédiés par les souverains suivants ne laissent guère de place au doute : une de leurs motivations est d'imiter Alexandre le Grand. Ce dernier offre un sacrifice à la déesse conformément à un oracle, et lui dédie des bucranes et des armes. À sa suite, Ptolémée $\mathrm{I}^{\text {er }}$ offre un sacrifice à la déesse et lui offre les crânes des victimes. Pyrrhus l'imite le plus fidèlement : il sacrifie également suite à un oracle et offre des bucranes et des armes. Les rois Hiéron et Philippe V dédient des armes.

Mais d'autres motivations interviennent sans doute: Pyrrhus indique par ces dédicaces un intérêt pour le bassin oriental de la Méditerranée. Hiéron, quant à lui, vise sans doute davantage un public sicéliote. En effet, lui-même originaire de Syracuse, une colonie corinthienne, il se positionne "positivement » par rapport aux cités filles de Lindos en offrant des armes à Athéna Lindia : il rend hommage à celle qui était la divinité poliade de leur métropole.

Ce groupe d'étrangers présente un contraste frappant avec celui de la période archaïque. En effet, ce n'est pas l'activité de Lindos qui motive les dédicants, mais son renom passé et celui de son sanctuaire, ainsi que des raisons «personnelles», ce qui n'empêche pas Lindos de récupérer le prestige de ces dédicants à son compte.

\section{c) Les prêtres d'Athéna Lindia}

Les deux premières dédicaces royales, celles d'Alexandre le Grand et de Ptolémée $\mathrm{I}^{\mathrm{er} 44}$, sont les seules de la chronique à être datées selon le système lindien, par le prêtre éponyme d'Athéna. Selon l'édition de F. Jacoby, suivi

richesses dans les relations entre le souverain, la "Maison du roi" et les savants de cour : un état des lieux », in V. CHANKOwski et F. DuYRAT (éds), Le roi et l'économie. Autonomies locales et structures royales dans l'économie de l'empire séleucide. Topoi suppl. 6 (2004), p. 208, n. 71.

42 Diodore, XX, 100.

${ }^{43}$ Blinkenberg, o.c. (n. 1), 194. Sur le contexte, H.U. WiEmer, Krieg, Handel und Piraterie. Untersuchungen zur Geschichte des hellenistischen Rhodos, Berlin, 2002 (Klio Beihefte. Neue Folge Bd 6), p. 62-64; les Rhodiens ont fait appel à Alexandre pour les libérer du joug perse. L'auteur, qui mentionne la dédicace à Athéna, n'en propose pas d'interprétation.

${ }^{44} \mathrm{~N}^{\circ} 38, \mathrm{C} 1.107$ et $\mathrm{n}^{\circ} 39$, C. 1. 112. Sur l'identification du roi lagide, voir HigBIE, o.c. (n. 1), p. 137 et WiEMER, o.c. (n. 43), p. 92 
par C. Higbie ${ }^{45}$, cette datation figurait dans le texte de la dédicace. D'autres attestations de dédicaces royales, par Alexandre ou par d'autres souverains du III $^{\mathrm{e}}$ siècle, paraissent exclure cette hypothèse ${ }^{46}$. Plus vraisemblablement, les auteurs de la chronique ont trouvé cette précision dans leur source, les archives des Lindiens, mais elle n'a jamais figuré dans les dédicaces. Il faudrait donc en revoir l'édition et refermer les guillemets avant la mention des prêtres éponymes ${ }^{47}$.

Dans les deuxième et troisième récits d'épiphanie, la déesse apparaît en rêve à un prêtre et à un ancien prêtre, tous deux nommés, et agit par leur entremise. Ces personnages jouent un rôle déterminant d'intermédiaire entre la déesse et les Lindiens (ou les Rhodiens selon le cas). Les prêtres d'Athéna, dont la fonction est éponyme, occupent une place très élevée dans la société lindienne à l'époque hellénistique ${ }^{48}$. Rhodes et ses dèmes comptent parmi les rares structures civiques grecques où les prêtrises sont hiérarchisées et organisées en cursus. À Lindos, le sommet de la progression est naturellement le service à Athéna, l'étape suivante étant la prêtrise d'Hélios dans la cité de Rhodes. La prêtrise d'Athéna ne peut sans doute être exercée qu'une fois, mais les listes de prêtres montrent que malgré cette restriction la fonction fut monopolisée par quelques familles.

L'évocation récurrente de prêtres dans la Chronique n'est guère étonnante. Elle est d'ailleurs concentrée à la période où la prêtrise acquiert de l'importance, sans doute après le synécisme rhodien. Dans la première épiphanie, en effet, la seule qui antédate sûrement le synécisme et l'incendie du temple, Athéna apparaît à un archontos (D 1. 13-4). Les épiphanies, bien plus que les dédicaces, servent à mettre en évidence le rôle des prêtres. Cependant, la datation de la dédicace d'Alexandre (et dans une moindre mesure celle de Ptolémée $\mathrm{I}^{\mathrm{er}}$ ) permet d'associer un prêtre au dédicant le plus prestigieux de la dernière période. Ces mentions rappellent la présence physique du sanctuaire et de son personnel, porte-parole essentiel de la déesse.

\section{d) Conclusions}

Les notices de la troisième période révèlent la passivité politique de Lindos après son intégration dans la cité de Rhodes : en tant que dème, la communauté ne peut plus mener une politique internationale propre. Cette période est caractérisée en revanche par une importance croissante de la déesse, ou

\footnotetext{
45532 F C 1.107 et 112 (éd. JACOBY); HigBie, o.c. (n. 1), p. 40, 182.

46 On peut citer tant le texte de dédicaces par Alexandre le Grand [par ex. SEG 30 (1980), 1533; P.J. Rhodes et R. OsBorne, Greek Historical Inscriptions 404-323, Oxford, 2003, n 86] que par d'autres souverains. Voir par ex. à Délos, F. Durrbach, Choix d'inscriptions de Délos, Paris, $1921, \mathrm{n}^{\circ} 35,36,51,52,53,56,57$

${ }^{47}$ Cette hypothèse confirmerait aussi que les deux auteurs de la Chronique se sont fondés sur des sources, sans s'intéresser beaucoup aux objets. Voir SHAYA, l.c. (n. 2), p. 428, ce qu'elle contredit à la n. 25.

${ }^{48}$ Voir GABrielsen, o.c. (n. 19), p. 30-31, 116-118, 130-134, n. 95 p. 207, etc. B. Dignas, «Rhodian Priests after the Synoecism », AncSoc 33 (2003), p. 35-51.
} 
plutôt de ses prêtres. Lorsqu'une action locale motive une dédicace, elle est de nature religieuse : une épiphanie de la déesse, un oracle. De même, dans les récits de cette période qui évoquent la «politique extérieure » de Rhodes, c'est l'intervention d'un dieu qui permet aux Lindiens (et surtout au personnel du sanctuaire) de jouer un rôle décisif et d'assurer le salut de Rhodes ${ }^{49}$.

L'émergence d'un sanctuaire qui concentre l'énergie des Lindiens et la multiplication des récits dissimulent une réalité moins glorieuse. La répartition chronologique des notices et des épiphanies révèle en effet une concentration forte au $\mathrm{IV}^{\mathrm{e}}$ et au $\mathrm{III}^{\mathrm{e}}$ siècle. Il reste sur la pierre les traces de deux ou trois dédicaces supplémentaires. Mais quand bien même dateraient-elles toutes du II ${ }^{e}$ siècle, celles du début de l'époque hellénistique seraient toujours bien plus nombreuses. Cette répartition n'est pas un hasard, mais reflète le déclin du sanctuaire, attesté également par la documentation épigraphique. C'est bien le passé glorieux que met en évidence ce document, devenu un souvenir à l'époque des auteurs. Enfin, les notices de cette période, bien plus que celles relatives à des dédicaces plus anciennes, reflètent le contexte culturel dans lequel évoluent les auteurs : les valeurs et les modes de pensée de l'époque hellénistique se traduisent notamment par la grande importance donnée à la reconnaissance royale.

\section{Conclusions}

Au coeur du document rédigé par Tharsagoras et Timachidas se situe le culte d'Athéna Lindia. Son importance est révélée dans deux catalogues complémentaires : celui des dédicants venus l'honorer et celui de ses épiphanies. Dans le premier, chaque notice relate un acte de reconnaissance à Athéna Lindia, en citant la dédicace gravée sur les objets, une manière d'insister sur cet acte religieux en hommage à une déesse dont le nom est mentionné dans la plupart des inscriptions ${ }^{50}$. La seconde partie, consacrée aux épiphanies, relate des interventions actives de la divinité dans la vie des Lindiens. En sélectionnant les dédicants et en imposant une organisation aux notices, les auteurs leur ont cependant donné un sens qui dépasse la simple énumération.

Deux axes, l'un temporel l'autre géographique, structurent l'œuvre. L'organisation chronologique globale du document met l'accent sur la longue durée du culte d'Athéna qui remonte à une époque mythique, antérieure même à la Guerre de Troie. Le découpage du temps à l'intérieur du catalogue trahit toutefois des préoccupations d'ordre historique, révélant l'imbrication étroite entre la vie du sanctuaire et celle de la cité ou du dème qui l'abrite. Les grandes périodes de l'histoire de Lindos déterminent une division en trois parties à l'intérieur desquelles la répartition géographique permet d'insister

${ }^{49}$ Voir le commentaire de B. Dignas sur la 3 épiphanie, DignAs, l.c. (n. 48), p. 44-45.

50 En raison de la nature des notices, ce document a été comparé aux « inventaires de temples »: B. Dignas, "Inventories' or 'Offering lists'? Assessing the Wealth of Apollo Didymaeus' ", ZPE 138 (2002), p. 240-241. Mais ce rapprochement est un peu factice, fondé seulement sur la mention de dédicaces dans tous ces textes. La fonction de ces documents est en effet très différente. 
d'abord sur le caractère local et poliade de la divinité, et ensuite sur son aura internationale. À l'époque mythique, comme à l'époque hellénistique, périodes où la Cité-État de Lindos soit n'existe pas encore, soit n'existe plus, les dédicants non-lindiens dominent largement, soulignant avec acuité le prestige du culte d'Athéna. La plupart jouissent d'ailleurs d'un statut particulier : héros, demi-dieux, souverains, ils peuvent faire eux-mêmes l'objet d'un culte. Ils se situent donc à l'intermédiaire entre l'humain et le divin et leur hommage à Athéna Lindia fait ressortir d'autant mieux sa puissance et sa renommée.

L'époque archaïque fait exception à ce schéma : à cette période, le faite de l'histoire lindienne, le plus grand nombre de Lindiens sont nommés comme dédicants (9 notices contre 10 pour des étrangers). Lindos apparaît alors comme une cité dynamique, qui mène des entreprises militaires et coloniales, et développe un large réseau commercial et diplomatique. Les dédicants étrangers appartiennent, pour l'essentiel, au réseau colonial de Lindos; leur liste ne se comprend que du point de vue de la cité. Les deux exceptions relèvent précisément d'autres critères : le don d'Amasis est mis en évidence parce qu'il est célèbre à travers le monde grec et donc, dans une perspective hellénistique, rehausse le prestige du sanctuaire; les relations avec les Perses servent à inscrire Lindos dans une histoire grecque.

À l'époque hellénistique, ou plutôt après le synécisme, pour l'exprimer en termes lindiens, la position du dème par rapport à la cité de Rhodes devient la préoccupation principale: il s'agit de ne perdre ni son identité, ni son importance. C'est l'époque où les épiphanies de la déesse, déjà mises en place par un « coup d'essai » dans un épisode sans doute fictif, se multiplient et épaulent les prétentions des prêtres, leur offrant l'occasion de jouer un rôle à l'échelle de la cité rhodienne. Grâce à ce thème, les auteurs peuvent développer leur propos sur l'importance du sanctuaire à cette époque. En revanche, les apparitions ne semblent pas susciter d'intérêt en-dehors de l'île (du moins dans les récits conservés) et n'attirent pas de visiteurs. ${ }^{51}$ Le prestige du sanctuaire vient d'une autre source à cette période : les dédicaces des rois, qui révèlent son renom international. Mais cette liste réduit le sanctuaire à un lieu de publicité, où les souverains pour des raisons variées, mais non motivées par une action du dème de Lindos, viennent se mettre en avant.

De l'époque archaïque à l'époque hellénistique, les modes de construction de l'identité et du prestige de Lindos changent : les activités militaires et coloniales, dominantes à haute époque, cèdent la place au religieux. Ce changement est aussi attesté par d'autres sources. Ainsi, un document daté de la fin du $\mathrm{IV}^{\mathrm{e}}$ siècle, à mettre en relation donc avec la $3^{\mathrm{e}}$ partie du catalogue, conserve le souvenir d'une victoire juridique du dème : Lindos a en effet obtenu que, malgré le synécisme, les prêtrises lindiennes et l'accès aux cultes locaux

\footnotetext{
51 Au sujet des épiphanies, SHAYA, l.c. (n. 1), p. 433-4 a une interprétation davantage centrée sur le texte lui-même.
} 
soient réservés aux habitants du dème ${ }^{52}$. De telles restrictions sont attestées ailleurs: Olymos, après son intégration à la cité de Mylasa par sympolitie, continue néanmoins à contrôler l'accès à ses cultes et à ses prêtrises ${ }^{53}$. L'identité locale est désormais préservée surtout grâce à un rapport privilégié aux cultes. L'importance croissante du personnel des sanctuaires de Lindos et la hiérarchie établie entre les diverses prêtrises sont un corollaire de ce repli : l'identité sociale des Lindiens se concentre désormais aussi dans le domaine du religieux.

Au terme de cette analyse, on comprend mieux les choix de Tharsagoras et Timachidas : critères de sélection, organisation,... tout doit contribuer à la glorification d'Athéna Lindia. L'ancienneté de son culte, son large rayonnement, le rôle de la déesse dans le salut de la cité rhodienne, témoignent de sa puissance et de l'éclat de son culte. La rédaction de la "Chronique » constitue un acte religieux décidé par les Lindiens à une époque où, si l'on en croit la répartition chronologique des dernières dédicaces, même l'aura du sanctuaire décline. La stèle et le texte qui s'y trouve gravé forment un tout, un monument en l'honneur d'Athéna, qui rappelle combien le sanctuaire a brillé à travers les siècles et, par sa propre existence, le fait briller une fois encore. L'œuvre s'adresse à un public local : c'est l'identité lindienne qui est en jeu, une identité définie toutefois aussi par rapport aux voisins immédiats, habitants d'Ialysos ou de Camiros. Cette ouvre rappelle en effet le rôle de Lindos et surtout d'Athéna Lindia au sein de la cité rhodienne, une place que seuls les autres dèmes de la cité pourraient lui disputer.

Centre de recherche sur la Cité grecque - CP 175/01

Natacha MASSAR

Université libre de Bruxelles

50, av. F.D. Roosevelt

B - 1050 Bruxelles

Courriel : nmassar@ulb.ac.be

52 IG XII 1, 761 (Syll $\left.l^{3}, 340\right)$, 1. 38-43. Voir DignAs, l.c. (n. 47), p. $45-46$ qui suggère qu'il s'agissait aussi d'interdire l'accès aux habitants de la Pérée rhodienne récemment intégrés au dème de Lindos.

53 Voir G. REgER, « Sympoliteia in Hellenistic Asia Minor », in S. COLvin (éd.), The GrecoRoman East. Politics, Culture, Society, Cambridge, 2004 (Yale Classical Studies 31), p. 166-168 qui fait le rapprochement avec le cas lindien. 